\title{
Tecnologías de la información y comunicaciones en el proceso enseńanza-aprendizaje universitario: Progreso educativo
}

Information and communications technologies in the university teaching-learning process: educational progress

Recibido: mayo 16 de 2019 | Revisado: junio 14 de 2019 | Aceptado: julio 11 de 2019

\author{
Paulina E. Yarasca Carlos ${ }^{\text {ia }}$ \\ George Argota PÉrez ${ }^{2, *}$ \\ Doris LaOs ANCHANTE ${ }^{\text {IB }}$ \\ Jorge García CeCCarelli ${ }^{\mathrm{IB}}$ \\ Rosa G. Castillo Paredes ${ }^{\text {ic }}$ \\ Patricia P. Huarancca Contreras ${ }^{\text {ID }}$
}

\begin{abstract}
Resumen
El propósito del trabajo fue analizar las tecnologías de la información y comunicaciones en el proceso enseñanzaaprendizaje universitario como progreso educativo. Se indicó cómo se acrecienta el conocimiento tanto en los docentes y alumnos mediante el uso aplicativo de las tecnologías de la información y comunicaciones (TIC). Asimismo, se destacó la necesidad de los docentes en mantener capacitaciones de forma sistemáticas para el uso de las TIC en aula y lo que representa como mecanismo de integración para la formación profesional. Finalmente, se describe la creación de habilidades binomiales entre docentes-estudiantes que posibilitan modificar el enfoque tradicional por enfoque de competencia durante la nueva enseñanza del siglo XXI..
\end{abstract}

Palabras clave: enseñanza-aprendizaje, formación, situación numérica, nivel universitario

\begin{abstract}
The purpose of the study was to analyze the information and communications technologies in the teachinglearning process as educational progress. It was indicated how knowledge is grown in both teachers and students through the application of information and communication technologies (ICT). It also highlighted the need of teachers to maintain systematic training for the use of ICT in the classroom and what it represents as an integration mechanism for vocational training. Finally, it describes the creation of binomial skills among teachers-students that make it possible to modify the traditional approach by competence approach during the new teaching of the 21 st century.
\end{abstract}

Key words: teaching-learning, training, numerical situation, university level

\footnotetext{
(C) Los autores. Este artículo es publicado por la Revista Campus de la Facultad de Ingeniería y Arquitectura de la Universidad de San Martín de Porres. Este artículo se distribuye en los términos de la Licencia Creative Commons Atribución No-comercial - Compartir-Igual 4.0 Internacional (https://creativecommons.org/licenses/ CC-BY), que permite el uso no comercial, distribución y reproducción en cualquier medio siempre que la obra original sea debidamente citada. Para uso comercial contactar a: revistacampus@usmp.pe.
} 


\section{Introducción}

El empleo de las Tecnologías de la Información (TIC) permite regenerar el conocimiento y su interpretación tanto en el proceso del aprendizaje como en el del futuro desempeño profesional de forma eficiente (Tikhonova \& Tereshkova, 2014; Khromovetal., 2015). El mejoramiento de las TIC garantiza cambios significativos en la enseñanza, crea nuevos retos de enfoques en la enseñanza y el aprendizaje que exigen sustituir a los modelos tradicionales (Klimova, 2015) y que aparezcan informaciones en línea; es decir, el acceso abierto una vez que sean presentados los conocimientos lo cual, indica que puedan ser reutilizados y adaptados (Al-Rahmi et al., 2019). Asimismo, las TIC permiten de forma eficiente promover el desarrollo interpretativo del conocimiento (Ezugwu et al., 2016), ya que generan tendencias metodológicas durante los estudios e investigaciones (Ebben \& Murphy, 2014; Veletsianos \& Shepherdson, 2015; Raffaghelli, Cucchiara \& Persico, 2015; Deng \& Benckendorff, 2017).

Un aspecto clave en el proceso de enseñanza-aprendizaje en el nivel universitario, es que los docentes valoren a sus estudiantes como verdaderos clientes (Bridges, 1999; Obermiller \& Atwood, 2011), aunque se señala que, debe evitarse la dirección en torno a la popularidad o entretenimiento durante las clases ante el criterio arbitrario de grupos (Gross \& Hogler, 2005; Acevedo, 2011). Es por ello que, debe mantener una relación profesional con los estudiantes y no, su condicionamiento en ambientes físicos (Bailey, 2000; Armstrong, 2003). El aspecto indispensable en la actualidad para evitar distorsión son las TIC, pues las mismas finalmente, crean oportunidades, mejoran la calidad de la educación, además, de propiciar habilidades debido a, la motivación que se genera por parte de los instructores (Ahmadi, Keshavarzi \& Foroutan, 2011; Yakovleva \& Goltsova, 2016).

\section{Desarrollo}

$\mathrm{Si}$ bien es cierto que, en ocasiones las TIC no desarrollan algunas habilidades como el aprendizaje presencial, tampoco significa que sean herramientas excluyentes, por el contrario, revelan ser tecnologías complementarias (Brinson, 2015; Alves et al., 2016). En la actualidad, el trabajo en grupos o equipos requiere de tiempo para la discusión de las ideas, ya que todo aprendizaje es un proceso social (Corter et al., 2011) pero el uso de las TIC de manera simultánea, garantiza determinados resultados en el aprendizaje (Fidalgo et al., 2014; Marques et al., 2014).

En ese contexto, cabe señalar que el manejo de las TIC, fuera del campus tradicional, mitiga las incertidumbres de las prácticas habituales en colectivo (Alves et al., 2016) y para aquellas personas con habilidades diferentes (discapacidad) posibilitan acceder al intercambio de información (Heradio et al., 2016). Indiscutiblemente, el desarrollo tecnológico en primer lugar, apoya nuevas formas de enseñanza y aprendizaje (Drent \& Meelissen, 2008) y en segundo orden supone que los docentes deben desarrollar alfabetización sobre la tecnología en sus alumnos para prepararlos en afrontar las demandas del presente siglo XXI (ISTE, 2008; UNESCO, 2011).

Lo planteado sugiere, según Sang et al., (2010) y Agyei \& Voogt (2011) que 
la educación debe ser educada para el uso de las tecnologías como instrumentos de sustento para formar a los estudiantes. El uso innovativo de las TIC, aún no cubre las expectativas, pues la incorporación por parte de los docentes en sus clases es limitada, además, el nivel deaplicación varía de un escenario a otro. Asimismo, deberá reconocerse que, las TIC están para apoyar las orientaciones y no, como un medio de transformar las prácticas pedagógicas (Tondeur et al., 2013). Aunque se está expandiendo la investigación sobre la enseñanza de docentes con tecnología en la formación propiamente docente, todavía permanece distante de la investigación sobre enseñanza y aprendizaje con tecnologías (Ottenbreit-Leftwich et al., 2010; Tømte et al., 2015).

\section{Aplicaciones}

En un estudio sobre la percepción de la competencia en el aprendizaje de por vida de los maestros y sus habilidades en el uso de las tecnologías de la información y la comunicación donde fueron seleccionados de forma aleatoria 102 maestros se indicó la necesidad de organizar actividades como seminarios, conferencias y paneles para desarrollar la ciencia en los docentes como personas que deban aprender el uso de las TIC durante toda su vida laboral. Se halló que, si bien fue cierto el buen reporte de usar las TIC, las habilidades estuvieron muy por debajo en el grupo de análisis siendo una necesidad de aprender constantemente las habiliades de las TIC para mantener buena competencia profesional (Ozdamli \& Ozdal, 2015). Otro estudio indicó que, si bien los maestros promueven el aprendizaje de por vida en los estudiantes, les cuesta dificultad apoyarlos continuamente (Klug et al., 2014). Es posible que una de las principales causas radique en limitaciones para el manejo de las propias TIC, cuales comprenden desde herramientas visuales, de audio e impresas para alcanzar, diseñar y desarrollar informaciones relevantes en el proceso de enseñanza-aprendizaje (Gorghiu et al., 2013).

Del mismo modo, la aplicación de las TIC se ha promovido para implementarse en las conferencias durante el proceso de enseñanza-aprendizaje, pero sigue reconociéndose los grandes desafíos que aún están por enfrentarse. El uso de las TIC en el proceso de la enseñanza mejora el conocimiento y crea oportunidades en el campo de la interpretación donde Tikhonova \& Tereshkova (2014) informaron bajo un enfoque por competencia el logro de aspectos lingüísticos y culturales durante el aprendizaje de la lengua china, además, en la enseńanza de otras lenguas desde su nivel avanzado con fines como especializaciones en economía y finanzas durante el aprendizaje del idioma ruso como lo señaló Khromov et al., (2015).

El mejoramiento de nuevas TIC emergentes ha motivado el cambio de los enfoques de la enseńanza y el aprendizaje el mismo que es explorado en tres aristas diferentes: el alumno, la interacción alumno-alumno y alumnodocente, así como la movilidad desde el enfoque tradicional a la competencia donde la búsqueda de metodologías continua la exploración para entender el comportamiento de transición ante la interpretación de fenómenos y que puede motivar a la comunidad de investigación como se muestra en la Figura 1, según Klimova (2015). 


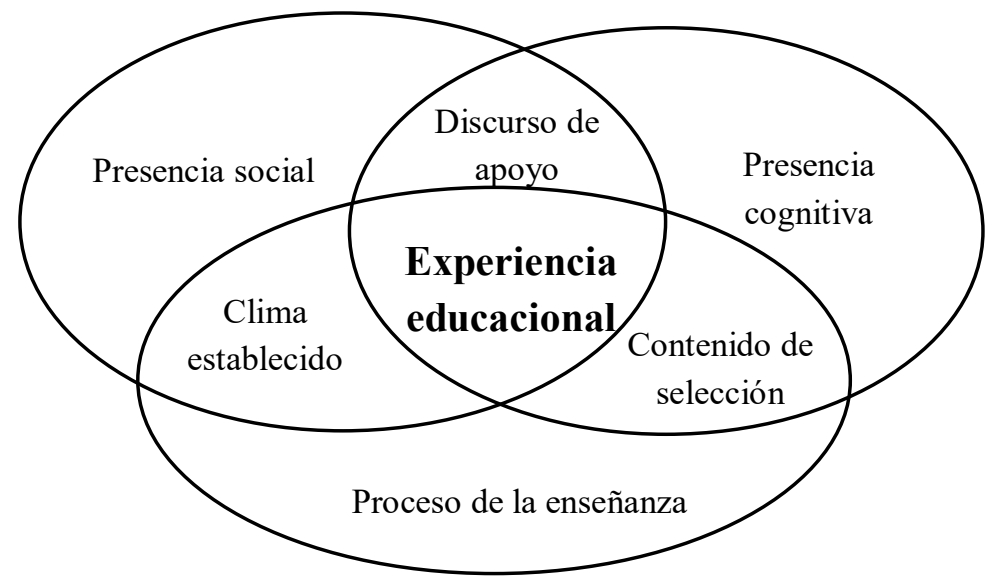

Figura 1. Modelo de comunidad de integración

\section{Conclusiones}

Finalmente, las TIC están siendo evaluadas durante programas de necesidad mediante talleres de capacitación de forma permanente donde modelos de diseños permiten su análisis. Lucas et al., (2017) valoraron: 1 ro) diseńo del taller, 2do) calidad del contenido del taller, 3ro) calidad de la entrega del contenido del taller y 4to) relevancia del taller.
Un aspecto clave se centra en las expectativas de los participantes sobre el crecimiento de sus conocimientos sobre el posible aprendizaje de las TIC. Asimismo, los autores proponen un índice de evaluación del taller (WEI) para evaluar el desempeńo, realizar un seguimiento de la mejora en el tiempo y desarrollar estrategias encaminadas a la incorporación de nuevos aspectos en la capacitación docente.

\section{Referencias}

Acevedo, A. (2011). Business ethics 101: the student is not a customer. InternationalJournal of Management Education; 9(3), 1-11. https:// aom.org/Publications/AMLE/ Business-Ethics-and-ManagementEducation.aspx

Agyei, D.D. \&Voogt, J. (2011). Exploring the potential of the will, skill, tool model in Ghana: Predicting prospective and practicing teachers' use of technology. Computers \& Education; 56(1), 91-100. https://doi.org/10.1016/j. compedu.2010.08.017
Ahmadi, S., Keshavarzi, A. \& Foroutan, M. (2011). The application of information and communication technologies (ICT) and its relationship with improvement in teaching and learning. ProcediaSocial and Behavioral Sciences; 28, 475-480. https://doi. org/10.1016/j.sbspro.2011.11.091

Al-Rahmi, W., Aldraiweesh, A., Yahaya, N., Bin, K.Y., Zeki, A.M. (2019). Massive open online courses (MOOCs): Data on higher education. Data in Brief; 22, 118125. https://doi.org/10.1016/j. dib.2018.11.139 
Alves, G., Viegas, C., Lima, N. \& Gustavsson, I. (2016). Simultaneous usage of methods for the development of experimental competences. International Journal of Human Capital and Information Technology Professionals; 7(1), 54-73. http://doi.org/10.4018/ IJHCITP.2016010104

Armstrong, M. (2003). Students as clients: a professional services model for business education. Academy of Management Learning and Education; 2, 371-374. https://doi. org/10.5465/amle.2003.11901964

Bailey, J.J. (2000). Students as clients in a professional/client relationship. Journal of Management Education; 24, 353-365. https://doi.org/10.1 177\%2F105256290002400306

Bridges, E. (1999). Experiential learning and customer needs in the undergraduate marketing research course. Journal of Marketing Education; 21(1), 51-59. https://doi. org/10.1177\%2F0273475399211007

Brinson, J.R. (2015). Learning outcomes achievements in non-traditional (virtual and remote) versus traditional (hands-on) laboratories: A review of the empirical research. Computers \& Education; 87, 218237. http://dx.doi.org/10.1016/j. compedu.2015.07.003

Corter, J.E., Esche, S.K., Chassapis, C., Ma, J., \& Nickerson, J.V. (2011). Process and learning outcomes from remotelyoperated, simulated, and handson student laboratories. Computers
\& Education; 57(3), 2054-2067. http://dx.doi.org/10.1016/j. compedu.2011.04.009

Deng, R. \& Benckendorff, P. (2017). A contemporary review of research methods adopted to understand students' and instructors' use of Massive Open Online Courses (MOOCs). International Journal of Information and Education Technology: 7(8), 601-607. https://doi.org/10.18178/ ijiet.2017.7.8.939

Drent, M. \& Meelissen, M. (2008). Which factors obstruct or stimulate teacher educators to use ICT innovatively? Computers \& Education; 51(1), 187-199. https://doi.org/10.1016/j. compedu.2007.05.001

Ebben, M. \& Murphy, J.S. (2014). Unpacking MOOC scholarly discourse: A review of nascent MOOC scholarship. Learning, Media and Technology; 39(3), 328345. http://dx.doi.org/10.1080/17 439884.2013 .878352

Ezugwu, A.E., Ofem, P.O., Rathod, P., Agushaka, J.O. \& Haruna, S. (2016). An Empirical Evaluation of the Role of Information and Communication Technology in Advancement of Teaching and Learning. Procedia Computer Science; 92, 568-577. https://doi. org/10.1016/j.procs.2016.07.384

Fidalgo, A., Alves, G., Marques, A., Viegas, C., Costa-Lobo, C. \& et al. (2014). Adapting Remote Labs to Learning Scenarios: Case Studies 
Using VISIR and RemotElectLab. IEEE Revista Iberoamericana de Tecnologias del Aprendizage; 9(1), 33-39. https://doi.org/10.1109/ RITA.2014.2302071

Gorghiu, M.L., Gorghiu, G., Dogan, Y.Z. \& Gerceker, P. (2012). Promoting the nano-technology concepts in secondary science education through ICT tools - the Romanian and Turkish teachers' perceptions. Global Journal on Technology; 3, 577-583.

Gross, M.A. \& Hogler, R. (2005). What the shadow knows: exploring the hidden dimensions of the consumer metaphor in management education. Journal of Management Education; 29, 3-16. https://doi. org/10.1177\%2F1052562903260034

Heradio, R., de la Torre, L., Galan, D., Cabrerizo, F., Herrera-Viedma, E. \& Dormido, S. (2016). Virtual and Remote Labs in Education: A Bibliometric Analysis. Computers in Education; 98; 14-38. http:// doi/10.1016/j.compedu.2016.03.010

International Society for Technology in Education: ISTE. (2008). ISTE Standards for teachers. Retrieved from http://www.iste.org/standards/ iste-standards/standards-for-teachers

Khromov, S.S., Gulayeva, N.A., Zelenetskaya, I.S., Minakova, L.Y. \& Sheketera, A.L. (2015). An algorithm for the integration of information and communication technologies in teaching languages for special purposes (the example of Russian as a foreign language).
Procedia-Social and behavioral sciences; 200, 224-229. https://doi. org/10.1016/j.sbspro.2015.08.056

Klimova, B.F. (2015). Teaching and Learning Enhanced by Information and Communication Technologies. Procedia-Social and Behavioral Sciences; 186, 898-902. https://doi. org/10.1016/j.sbspro.2015.04.112

Klug, J., Krause, N., Schober, B., Finsterwald, M. \& Spiel, C. (2014). How do teachers promote their students' lifelong learning in class? Development and first application of the LLL Interview. Teaching and Teacher Education; 37(1), 119-129. http://dx.doi.org/10.1016\%2Fj. tate.2013.09.004

Lucas, R.I., Promentilla, M.A., Ubando, A., Tan, R.G., Aviso, K. \& Yu, K.D. (2017). An AHP-based evaluation method for teacher training workshop on information and communication technology. Evaluationandprogramplanning;63, 93-100. https://doi.org/10.1016/j. evalprogplan.2017.04.002

Marques, M.A., Viegas, M.C., CostaLobo, M.C., Fidalgo, A.V., Alves, G.R., Rocha, J.S. \& Gustavsson, I. (2014). How remote labs impact on course outcomes: Various practices using VISIR. IEEE Transactions on Education; 57(3), 151-159. https://doi.org/10.1109/ TE.2013.2284156

Obermiller, C. \& Atwood, A. (2011). In defence of the student as customer metaphor. International Journal of Management Education; 9(3), 13-16. 
Ottenbreit-Leftwich, A.T., Glazewski, K.D., Newby, T.J. \& Ertmer, P.A. (2010). Teacher value beliefs associated with using technology: Addressing professional and student needs. Computers \& Education; 55(3), 1321-1335. https://doi.org/10.1016/j. compedu.2010.06.002

Ozdamli, F. \& Ozdal, H. (2015). Life-long Learning Competence Perceptions of the teachers and abilities in using informationcommunication technologies. Procedia - Social and Behavioral Sciences; 182. 718 - 725. http://. doi:10.1016/j.sbspro.2015.04.819

Raffaghelli, J.E., Cucchiara, S. \& Persico, D. (2015). Methodological approaches in MOOC research: Retracing the myth of Proteus. British Journal of Educational Technology; 46(3), 488-509.https:// doi.org/10.1111/bjet.12279

Sang, G., Valcke, M., Van Braak, J. \& Tondeur, J. (2010). Student teachers' thinking processes and ICT integration: Predictors of prospective teaching behaviours with educational technology. Computers \& Education; 54(1), 103112. https://doi.org/10.1016/j. compedu.2009.07.010

Tikhonova, E.V. \& Tereshkova, N.S. (2014). Information and communication technologies in the teaching of interpreting. ProcediaSocial and Behavioral Sciences; 154, 534-538. https://doi. org/10.1016/j.sbspro.2014.10.181
Tømte, C., Enochsson, A.B., Buskqvist, U. \& Kårstein, A. (2015). Educating online student teachers to master professional digital competence: The TPACKframework goes online. Computers \& Education; 84, 26-35. https://doi.org/10.1016/j. compedu.2015.01.005

Tondeur, J., Kershaw, L.H., Vanderlinde, R. \& Van Braak, J. (2013). Getting inside the black box of technology integration in education: Teachers' stimulated recall of classroom observations. Australasian Journal of Educational Technology; 29(3), 434-444.

United Nations Educational, Scientific and Cultural Organization: UNESCO. (2011). UNESCO ICT Competency framework for teachers. Retrieved from http:// unesdoc.unesco.org/ images/0021/002134/213475E.pdf

Veletsianos, G. \& Shepherdson, P. (2015). Who studies MOOCs? Interdisciplinarity in MOOC research and its changes over time. International Review of Research in Open and Distributed Learning; 16(3), 1-17. https://files.eric. ed.gov/fulltext/EJ1067914.pdf

Yakovleva, Y.V. \& Goltsova, N.V. (2016). Information and communication technologies as a means of developing pupils' learning motivation in elementary school. Procedia-Social and Behavioral Sciences; 233, 428-432. https://doi. org/10.1016/j.sbspro.2016.10.179 
